\section{What information can we give to the patient about the risks of strabismus surgery} JA Bradbury

\section{Abstract}

I hope to report the type, incidence, and clinical outcomes of severe complications from strabismus surgery in the United Kingdom and to help in the discussions involved in the consent process. The main part of the talk will revolve around a BOSU (British Ophthalmic Survey Unit) investigation. Cases were identified prospectively between 1 September 2008 and 31 August 2010. Questionnaire data were requested at the time of the complication recognition and at 6 months' follow-up. Outcome was graded I-V, with a poor or very poor outcome meaning either loss of corrected visual acuity or primary position double vision. A total of 60 completed reports of adverse events and complications were received during the study period. During the same time, $\sim 24000$ strabismus surgeries were carried out in the United Kingdom, yielding an overall incidence of

Eye Department, Bradford Royal Infirmary, Bradford, West Yorkshire, UK

Correspondence: JA Bradbury, Eye Department, Bradford Roya Infirmary, Duckworth Lane, Bradford BD9 6RJ, West Yorkshire, UK.

Tel: +44 (0)12 7436 4806; Fax: +44 (0)12 7438 2414; E-mail: John.Bradbury@ bthft.nhs.uk

Received: 16 July 2014 Accepted in revised form: 1 December 2014 Published online: 9 January 2015
1 in 400 operations (95\% binomial confidence, 1 per 333-500 operations). The most commonly reported complication was perforation of the globe $(19(0.08 \%))$, followed by a suspected slipped muscle (16 $(0.067 \%))$, severe infection (14 $(0.06 \%))$, scleritis $(6(0.02 \%))$, and lost muscle $(5(0.02 \%))$. Overall, complications were reported in adults and children in equal numbers; however, scleritis was significantly more common in adults. A poor or very poor clinical outcome was recorded as 1 operation per 2400 . The study limitations are as follows: the denominator was extrapolated from the number of surgeries in England, and there was an almost certain underreporting of cases. Complications with the potential for a poor outcome are relatively common, but the final clinical outcome is good in the majority of cases. Eye (2015) 29, 252-257; doi:10.1038/eye.2014.316; published online 9 January 2015

\section{Introduction}

The incidence and outcomes described in this chapter are from a 2-year prospective survey of severe complications of strabismus surgery carried out by the Author through the British Ophthalmic Surveillance Unit ${ }^{1}$ and the available literature.

The incidence of severe complications of strabismus surgery found through severe complications of strabismus surgery BOSU study reports an incidence of 1 in 400 operations. The incidence per 10000 operations is shown in Figure 1. Globe perforation is the most commonly reported complication with almost an incidence of $1: 1000$, with retinal detachment and endophthalmitis being the least commonly reported complications. Anterior segment ischaemia was not a part of the BOSU study into severe complications of strabismus surgery, but France ${ }^{2}$ reports the incidence as $1: 13000$ operations.

We devised a five-point scale depending on the severity of outcome of the complication. This is outlined in Table 1. The number of complications in the five categories split into adults and children is shown in Table 2 with the $P$-values. Although there were more infections and globe perforations in children and more surgically induced necrotising scleritis (SINS) and lost muscle in adults, only SINS reached statistical significance.

When we look at the outcome, $16 \%$ of patients with a complication had a poor or very poor outcome. This means that 1 in 2400 patients who were operated on had a poor or very poor outcome. 


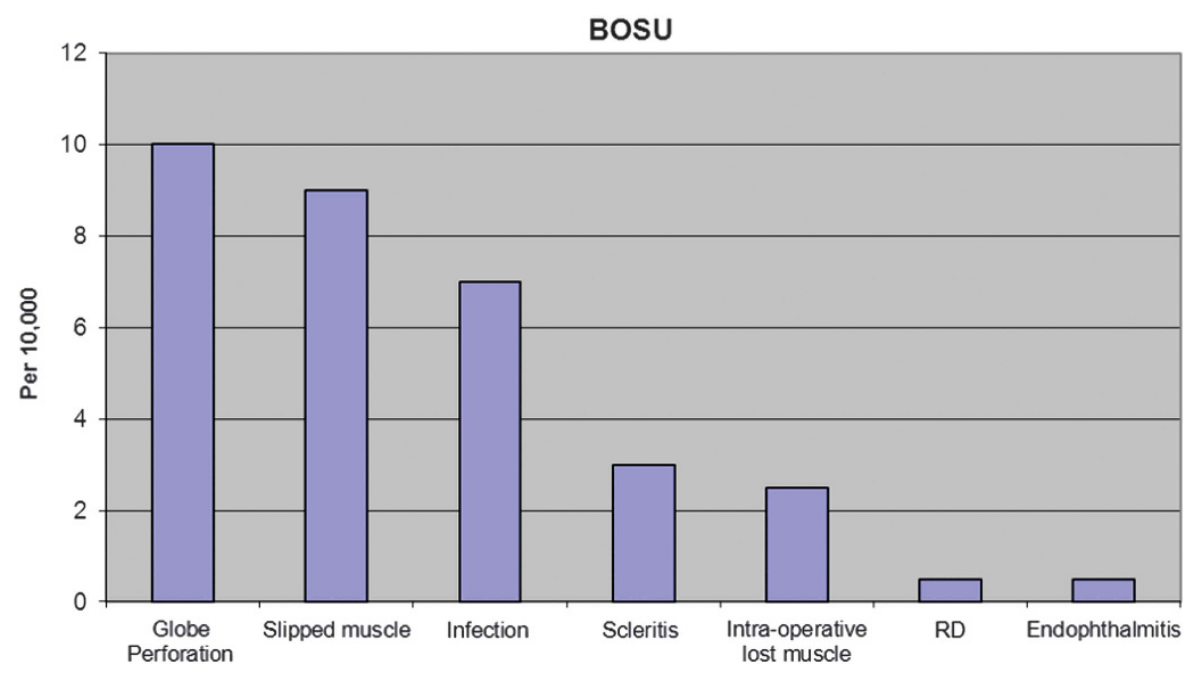

Figure 1 Incidence of complications per 10000 operations.

Table 1 Classification of outcome

\begin{tabular}{ll}
\hline Grade 1 & Good outcome \\
Grade 2 & Surgical or medical intervention good outcome \\
Grade 3 & Surgical or medical intervention asymptomatic but compromised outcome \\
Grade 4 & Surgical or medical intervention but poor outcome eg double vision or up to 2 lines of loss of vision on a Snellen chart \\
Grade 5 & Very poor outcome, greater than 2 lines of visual loss on a Snellen chart \\
\hline
\end{tabular}

Table 2 Complications in adults and children

\begin{tabular}{|c|c|c|c|c|c|c|c|c|}
\hline & Infection & SINS & Perforation & Lost muscle & Slipped muscle & Retinal detachment & Endophthalmitis & Total \\
\hline \multicolumn{9}{|l|}{ Adults } \\
\hline 1 & & & 1 & & & & & \\
\hline 2 & 2 & 3 & 2 & 1 & 7 & & & \\
\hline 3 & & & 4 & 3 & & 1 & & \\
\hline 4 & 1 & 2 & & 1 & & & & \\
\hline \multirow[t]{2}{*}{5} & & 1 & & & & & & \\
\hline & & & & & & & & 29 \\
\hline \multicolumn{9}{|l|}{ Children } \\
\hline 1 & & & 1 & & & & & \\
\hline 2 & 8 & & 10 & & 4 & & & \\
\hline 3 & 1 & & & & 4 & & & \\
\hline 4 & 2 & & & & 3 & & & \\
\hline 5 & & & 1 & & & & 1 & \\
\hline$P$-values & 0.0573 & 0.0312 & 0.3591 & 0.0625 & 0.4805 & 1.00 & 1.000 & 35 \\
\hline
\end{tabular}

Numbers exceed 60 as some patients had more than one complication.

I do not intend to discuss mild complications of strabismus surgery in this paper, but a list of such complications can be found in Table 3.

\section{Severe complications}

\section{Anterior segment ischaemia}

The incidence of significant anterior segment ischaemia has been estimated at 1:13000 cases. $^{2}$ This condition was not surveyed through the BOSU study, as it was felt by the BOSU panel that anterior segment ischaemia would be likely to be grossly unreported, especially in children in whom no regular slit-lamp examination takes place postoperatively. Severe outcomes are rare, although there is a report of someone developing phthisis bulbi. ${ }^{2}$ Severe complications of strabismus surgery are presented in Table 4.

Risk factors for the development of anterior segment ischaemia include increasing age, previous rectus 
surgery, and operations on multiple muscles in the same eye, especially on more than two recti muscles.

Symptoms and signs of anterior segment ischaemia can vary from mild uveitis and reduced iris profusion to a keratopathy. Lee ${ }^{3}$ suggested a four-grade classification (Table 5).

\section{Globe perforation}

Globe perforation is the most common severe complication of strabismus surgery. In the literature, it is reported in between 0.13 and $1 \%$ of cases. ${ }^{4,5}$ In the BOSU study on severe complications of strabismus surgery, the incidence was $1: 1000$. The incidence was more common in children, which may be because of surgical access, and only in one case was the outcome graded as poor or very poor. In this case, the patient developed endophthalmitis and had to have an evisceration. It therefore seems that although globe perforation is common a poor or very poor outcome is extremely rare.

The incidence may also vary in the type of surgery performed - for example, Faden procedures. Most cases of posterior segment perforation reported to BOSU had some form of peroperative treatment that usually

Table 3 Mild complications of strabismus surgery

\section{Dellen}

Chronic red eye

Ocular surface problems

Pyogenic granuloma

Tenons fascia prolapse

Epithelial inclusion cyst

Mydriasis

Reduced accommodation

Stitch granuloma

Chemosis

Haemorrhage

Table 4 Severe complications of strabismus surgery

Globe perforation
Orbital infection
Endophthalmitis
Surgical induced necrotising scleritis
Slipped muscle
Lost muscle
Retinal detachment
Adherence syndrome
Anterior segment ischaemia

Table 5 Classification of anterior segment ischaemia after Lee $^{3}$

\begin{tabular}{lcccc}
\hline Grade 1 & Grade 2 & Grade 3 & Grade 4 \\
\hline Reduced iris profusion & Pupillary abnormalities & Postoperative uveitis & Keratopathy \\
\hline
\end{tabular}

involved cryotherapy or laser. There was one case of retinal detachment in a high myope who had bilateral globe perforation from bilateral Harada-Ito procedure.

Rathod $^{6}$ in his survey of UK Ophthalmologists reported that $90 \%$ of cases with globe perforation were treated with cryotherapy and/or laser. In children, in whom globe perforation is more likely to occur, retinal detachment from a sharp globe perforation is unlikely to occur.

Strategies to prevent globe perforation would be to avoid suturing in areas that have obvious scleral thinning or to perform different techniques such as hang-back procedures.

\section{Orbital infection}

Orbital infection following strabismus surgery tends to take one of the two forms: diffuse orbital cellulitis or an abscess at the muscle insertion. In the BOSU study, there were 13 cases in total, and only 2 of these were in adult patients; the cases were split virtually 50/50 between diffuse orbital infection and muscle insertion abscesses. Three of the muscle insertion abscesses developed a slipped muscle and required surgical exploration to retrieve the muscle.

Kothari et $a l^{7}$ reported a similar case with bilateral orbital infection. Management of postoperative infection depends whether this is a diffuse orbital infection systemic antibiotics being curative in all cases reported or a muscle insertion abscess which if associated with a slipped muscle would require surgical exploration.

\section{SINS}

SINS is a rare but potentially serious complication of strabismus surgery. In the BOSU study, there were a total of six cases, all of which were in adults; there is one case in the literature that was reported to have occurred in a child. ${ }^{8}$ They were treated by a variety of medications including topical and systemic nonsteroidal and steroidal agents. One case had such severe scleritis that the patient required treatment with cyclophosphamide and developed posterior synechiae and a cataract, reducing the vision from 6/6 to 6/24; one-third of the cases had a poor or very poor outcome. The majority of patients were elderly, although two patients were in their 20s.

The evidence from the BOSU study would suggest that those patients developing SINS should perhaps be seen by a colleague with an interest in scleritis and uveitis who may be able to treat these patients. Also $\mathrm{O}^{\prime}$ Donoghue ${ }^{9}$ found a significant incidence of systemic 
disease in patients with SINS, and early diagnosis and aggressive treatment improved outcomes.

\section{Lost muscle}

In the BOSU study, a lost muscle was defined as a muscle lost peroperatively. There were a total of six lost muscles, five of which were in adults. The majority of these were elderly patients, four of whom had had a previous strabismus surgery. Looking at the muscles involved, four were medial recti and two were lateral recti. It has been previously reported ${ }^{10}$ that medial recti is probably the most common muscle that is lost at the time of surgery. All patients in the BOSU study had the muscles found peroperatively, and only one had a poor or very poor outcome.

Should a muscle be lost peroperatively, it is important to find the muscle rather than wait for any later date. If the muscle cannot be found, MacEwan et al ${ }^{10}$ suggests suturing the check ligaments and tenons that surround the muscle back to the original state, allowing some of the muscle action to be transferred, although these tissues belong to the eye. Some authors have advocated an orbital approach either for a posteriorly situated muscle through a lateral orbitotomy or transnasally. ${ }^{11}$

\section{Slipped muscle}

In the BOSU study, this was defined as the patient presenting with an overcorrection and a $50 \%$ or more reduction in movement in the action of a previously operated muscle. In the BOSU study, there were 18 cases, also slightly more common in children, but this did not reach statistical significance. In three of these cases, the slipped muscle was as a result of a muscle insertion infection postoperatively. Three out of $18 \mathrm{had}$ a poor or very poor outcome, and these were all in children.

Slipped muscles seem to take two main forms. One form is a true slipped muscle, similar to a lost muscle in that there was a problem with the sutures or insertion and soon after the operation the suture fails and the muscle slips, in which case one deals with this as one would with a lost muscle scenario. The more common presentation occurs many weeks or months or even years later with a gross limitation of action in the muscle that has slipped. An exploration often finds that the muscle is on a large pseudotendon-that is, the muscle is not attached to the sclera but is attached to the sclera via stretchy scar tissue.

\section{Endophthalmitis}

There was one case of endophthalmitis in the 2 years of the BOSU giving this was a 3-year-old who developed endophthalmitis following a globe perforation giving an incidence of $1: 24000$ cases. Treatment should follow current guidelines.

\section{Consent in Strabismus Surgery}

The second section of this paper discusses issues surrounding consenting patients for squint surgery and provide guidance to help you know how much to tell your patients and from whom you should seek consent.

\section{Consent is a process, not an event}

The act of placing a signature on a consent form should be the terminal event in a patient's journey from first appointment to eye theatre. The principle of taking consent before treatment is directed towards protecting a patient's autonomy. ${ }^{12}$ For consent to be valid, it must be given voluntarily by an adequately informed patient who has the capacity to consent. ${ }^{13}$ According to the Mental Capacity Act $2005^{14}$ (UK Law), capacity is defined by a person's ability to fulfil the four conditions set out below.
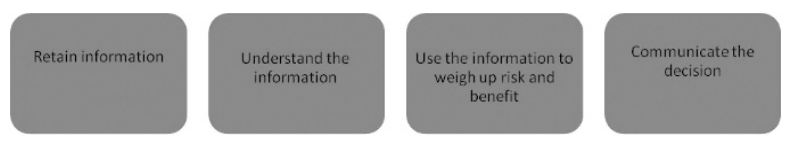

During the consent process, your patient develops a clear understanding of options, rationale, and outcomes for treatment. He or she can then weigh up risks and benefits to make an informed decision and develop reasonable expectations for surgical results.

It is considered best practice to document the discussions that you have with your patient at each stage of the consent journey and record what written information you have given them. In practice, I have found that the best approach is to provide adequate time for discussion in clinic and gain consent before the day of surgery. This allows your patient to reflect upon their decision, and any additional queries can be answered during confirmation of consent by the surgeon on the day of surgery. ${ }^{15}$ The American Medical Association recommends that consent should be performed by the physician providing the treatment. ${ }^{16}$ General Medical Council (GMC) guidelines state that you may delegate consent to another member of the healthcare team so long as they have sufficient knowledge of the procedure to describe risk and benefits and answer your patient's questions appropriately. ${ }^{17}$

\section{There are no rigid guidelines that set out what you must tell a patient}

Contrary to popular belief, there is no specific 'risk threshold' that mandates which complication you should 
or should not discuss with your patients. The legal standard describing what risks should be disclosed to the patient varies within and between countries. The standard of ethical care expected of healthcare professionals by their regulatory bodies may, in many cases, exceed the legal requirements. Generally, it is advisable to inform your patient of all significant possible adverse outcomes or unavoidable risks of surgery and make a record of the information given ${ }^{13}$ (see Table 6).

\section{Encourage a dialogue between you and your patient}

A careful balance needs to be struck between listening to what your patient wants and ensuring that they have sufficient information to make an informed decision. ${ }^{18}$ The complications discussed in the first half of this chapter will help inform this consent, and OMIC (Ophthalmic Mutual Insurance Company) ${ }^{19}$ considers the following common complications for strabismus surgery relevant to be discussed and recorded for each patient (see Table 7).

The duty lies with you as the surgeon to ascertain your patient's needs and wishes. Armed with this knowledge, you can then go on to discuss all factors that may be relevant to the individual, ${ }^{17}$ present information in a balanced way, and check that your patient has understood the information. For example, when operating on a patient's 'only eye', it would be relevant to discuss the small possibility of visual loss due to surgical complications; for a patient who depends on driving for

Table 6 Informed consent should cover these areas ${ }^{17,24}$

\section{Diagnosis}

Treatment options, including no treatment

Other possible procedures that may be necessary during surgery Common side effects/complications

Rare, significant complications

Anaesthetic options and risks

Table 7 Ophthalmic mutual insurance company informed consent for strabismus surgery ${ }^{19}$

Major risks of strabismus surgery

Need for additional surgeries.

Persistent misalignment, altered eyelid position, limitation

of eye movements, persistent visual problems.

Double vision.

Scar tissue formation.

Infection.

Haemorrhage or bleeding

Severe infection or bleeding leading to damage to the eye or rarely visual loss

Allergic reaction

Temporary side effects, such as corneal abrasion, conjunctivitis, eye ache. his job, the likelihood and duration of diplopia postoperatively will be especially relevant.

\section{Decision-making in children should be centred around shared responsibility between yourself and parents}

A minor is anyone under the age of 18 years (16 years in Scotland, and 19 years in some US states), and in both the United Kingdom and United States informed consent is required from a parent or legal guardian before medical treatment. Young individuals between the age of 16 and 18 years are presumed to be competent to consent in the United Kingdom: if they lack understanding or ability to consent, a parent can consent on their behalf. In most cases, a decision will be made that represents the best interests of the child. Although only one parent signature is required, it may be appropriate to involve both parents in decision-making.

Remember to consider an adolescent's developing capability for involvement in decision-making. ${ }^{20,21}$ A young person's ability to consent depends more upon their understanding of the nature of the proposed treatment and its possible consequences than upon their age. ${ }^{18,22}$ There is no simple criterion or measure of competence; your skill lies in assessing the young patient's competence in a particular context for a specific decision. ${ }^{23}$

In the United Kingdom, any young person under the age of 16 years must demonstrate their competence to consent, according to legal standards. In the United States, the ability of a young person between ages 12 and 18 years to consent is considered on a case-by-case basis.

I find it helpful to seek the child's assent, where developmentally appropriate, in addition to parental consent. This is considered good practice to help promote their empowerment in healthcare decision-making.

\section{Seeking consent in adults who lack capacity}

It is beyond the scope of this chapter to discuss consent in adults who are unable to consent for themselves. The legal approach to consent in an adult who may not be able to consent for themselves varies widely internationally. Individual cases will require different approaches, and it is important to seek advice from local regulatory bodies or medical defence organisation.

Case law is constantly evolving and varies internationally. Legal advice should always be sought if there is doubt as to the legal validity of a proposed intervention.

\section{Conflict of interest}

The author declares no conflict of interest. 


\section{References}

1 Bradbury JA, Taylor RH. Severe complications of strabismus surgery. J AAPOS 2013; 17(1): 59-63.

2 France T, Simon J. Anterior segment ischaemia syndrome following muscle surgery: the AAPOS experience. J Pediatr Ophthalmol Strabismus 1986; 23: 87-91.

3 Lee JP. Oliver anterior segment ischaemia. Eye 1990; 4: 1-6.

4 Noel LP, Bloom JN, Clarke WN, Bawazeer A. Retinal perforation in strabismus surgery. J Pediatr Ophthalmol Strabismus 1997; 34: 115-117.

5 Simon JW. Recognized scleral perforation during eye muscle surgery: incidence and sequelae. J Pediatr Ophthalmol Strabismus 1992; 29: 273-275.

6 Rathod D, Goyal R, Watts P. The management of globe perforation during strabismus surgery. J AAPOS 2010; 14: 25.

7 Kothari M, Sukri N. Bilateral Staphylococcus aureus sub-tenons abscess following strabismus surgery in a child. I AAPOS 2010; 14: 193-195.

8 Kearney FM, Blaikie AJ, Gole GA. Anterior necrotising scleritis and strabismus surgery in a child. J AAPOS 2007; 11: 197-198.

9 O'Donoghue E, Lightman S, Tuft S, Watson P. Surgically induced necrotising sclerokeratitis (SINS)-precipitating factors and response to treatment. Br J Ophthalmol 1992; 76: 17-21.

10 MacEwan C, Lee J, Fells P. Aetiology and management of the detached rectus muscle. $\mathrm{Br} J$ Ophthalmol 1992; 76 131-136.

11 Lenart T, Reichman O, MacMahon S. Retrieval lost medial rectus muscle with a combined ophthalmological and otolaryngo logic surgical approach. Am J Ophthalmol 2000; 130: 645-652.

12 Jackson E. Consent I: capacity and voluntariness. Medical Law: Text Cases and Materials. OUP: Oxford, 2006.

13 Department of Health. Reference guide to consent for examination or treatment, 2nd Edn, 2009. https://www.gov.uk/government/publications/referenceguide-to-consent-for-examination-or-treatment-secondedition.

14 Crown Copyright. Mental Capacity Act 2005. https:// www.gov.uk/government/uploads/system/uploads/ attachment_data/file/224660/ Mental_Capacity_Act_code_of_practice.pdf.

15 American Academy of Ophthalmology. Practice Guidelines for Informed Consent. http://one.aao.org/ Assets/9d4edb52-14e0-48d2-9e6c-7d5197c37958 / 634965436375930000/informed-consent-guidelines-posting12-19-11-final-pdf.

16 American Medical Association. Informed Consent. http:// www.ama-assn.org/ama/pub/physician-resources/ medical-ethics/code-medical-ethics/opinion808.page?

17 General Medical Council. Consent: patients and doctors making decisions together 2008. http:/ / www.gmc-uk.org/ static/documents/content/Consent_0510.pdf.

18 British Medical Association. Consent Toolkit. 5th Edition. http:/ / www.bma.org.uk/images/consenttoolkitdec2009_ tcm41-193139.pdf.

19 Ophthalmic Mutual Insurance Company (OMIC). Strabismus Surgery Consent Form. Version 4/16/09 ed 2009.

20 Committee on Bioethics. Informed consent, parental permission, and assent in pediatric practice. Pediatrics 1995; 95: 314-317.

21 American Academy of Pediatrics. AAP publications retired or reaffirmed. Pediatrics 2007; 119: 405.

22 General Medical Council. 0-18 years guidance 2007. http://www.gmc-uk.org/guidance/ethical_guidance/ children_guidance_index.asp.

23 Shaw M. Competence and consent to treatment in children and adolescents. Adv Psychiatr Treat 2001; 7: 150-159.

24 American Academy of Ophthalmology. Advisory Committee on the Code of Ethics: Informed Consent 2008. http://www.aao.org/about/ethics/upload/informedconsent-2008.pdf. 